\title{
Review \\ Singing Voice Detection: A Survey
}

\author{
Ramy Monir (D), Daniel Kostrzewa *(D) and Dariusz Mrozek
}

Department of Applied Informatics, Silesian University of Technology, 44-100 Gliwice, Poland; ramy.monir@polsl.pl (R.M.); dariusz.mrozek@polsl.pl (D.M.)

* Correspondence: daniel.kostrzewa@polsl.pl

check for updates

Citation: Monir, R.; Kostrzewa, D.; Mrozek, D. Singing Voice Detection: A Survey. Entropy 2022, 24, 114. https://doi.org/10.3390/e24010114

Academic Editor: Zoran H. Peric

Received: 19 November 2021

Accepted: 8 January 2022

Published: 12 January 2022

Publisher's Note: MDPI stays neutral with regard to jurisdictional claims in published maps and institutional affiliations.

Copyright: (c) 2022 by the authors. Licensee MDPI, Basel, Switzerland. This article is an open access article distributed under the terms and conditions of the Creative Commons Attribution (CC BY) license (https:/ / creativecommons.org/licenses/by/ $4.0 /)$.

\begin{abstract}
Singing voice detection or vocal detection is a classification task that determines whether there is a singing voice in a given audio segment. This process is a crucial preprocessing step that can be used to improve the performance of other tasks such as automatic lyrics alignment, singing melody transcription, singing voice separation, vocal melody extraction, and many more. This paper presents a survey on the techniques of singing voice detection with a deep focus on state-of-the-art algorithms such as convolutional LSTM and GRU-RNN. It illustrates a comparison between existing methods for singing voice detection, mainly based on the Jamendo and RWC datasets. Long-term recurrent convolutional networks have reached impressive results on public datasets. The main goal of the present paper is to investigate both classical and state-of-the-art approaches to singing voice detection.
\end{abstract}

Keywords: singing voice detection; vocal detection; music information retrieval; hidden Markov models; support vector machines; Mel-frequency cepstrum coefficients; perceptual linear prediction; short-time Fourier transform; deep learning models; datasets

\section{Introduction}

The singing voice is an essential component of music, serving as a communication channel for lyrics and rich emotions. A high level of expressiveness of human singing is even considered ideal for the instrument player to aspire toward. The human vocal apparatus generates sound by moving air forced by the diaphragm through the vocal folds, causing them to vibrate. Modulating airflow through the vibrating vocal folds produces a wealth of different timbres. Timbre is independent of the perceived pitch of a tone. It allows us to distinguish between vowels and consonants in words and the distinct sound qualities of various musical instruments.

Since human voice detection in music tracks is the basis for many advanced applications, it has been studied for several years. In the field of music information retrieval, singing voice detection (SVD) is the preprocessing step that can be used to improve the performance of other tasks such as automatic lyrics alignment [1-3], singing melody transcription [4,5], singing voice separation [6-8], vocal melody extraction [9], lyric transcription [10,11], singer identification [12], etc.

To the best of our knowledge, no recent review article has been written on the singing voice detection problem. As a result, in this paper, we would like to fill this gap, and we investigate the classical approaches of SVD systems [13] which focus on the acoustic similarity between singing voice and speech, using cepstral coefficients [13] and linear predictive coding [14]. In addition, we review the existing SVD systems with the use of machine learning classifiers such as random forests, artificial neural networks, and support vector machines combined with a large set of audio descriptors (e.g., spectral flatness) as well as special features such as fluctograms [15]. Lastly, we review the state-of-theart techniques using deep neural networks, which the SVD systems can apply to learn features using a recurrent neural network (RNN) [16] and convolutional neural network (CNN) [17]. Lately, new types of neural network structures have been widely applied to 
solve many difficult tasks [18]. There is a difference between a human singing voice and regular speech (speaking voice), mainly in intonation manipulation. Yasunori et al. [19] proposed two models to differentiate between a singing voice and a speaking voice based on Mel-frequency cepstrum coefficients (MFCCs). A singing voice utilizes vocal cord muscle tension to regulate the pitch and duration. Its average intensity is thus beyond that of speech, its dynamic vary is more significant, and its tone is usually totally different from that of speech [20].

In order to locate vocal segments, researchers usually extract one or more types of features from the audio signals and then use the classifier to detect them [18]. There are various types of attributes, but MFCCs and the spectrum obtained with short-time Fourier transform (STFT) were the most commonly used features for the SVD task. The features and statistical classification methods used in speech recognition have some limitations in detecting singing voices. Deep learning, with its powerful feature representation as well as time and space modeling capabilities, has recently begun to be used in singing voice detection [21].

To detect the singing voice in music tracks, researchers usually split the speech signal into three portions: voiced (a strong sound in which the vocal cords vibrate), unvoiced, and silent parts. There are several voiced and unvoiced regions in speech. If the system's input excitation is a nearly periodic impulse sequence, the corresponding speech appears visually nearly periodic and is referred to as voiced speech. While the excitation is random noise-like, the resulting speech signal is random noise-like as well, with no periodic nature, and is referred to as unvoiced speech. The classification of speech signals as voiced or unvoiced provides a preliminary acoustic segmentation for speech processing applications such as speech synthesis, speech enhancement, and speech recognition [22].

This paper is organized as follows: Section 2 is focused on feature extraction, and Section 3 presents the most used datasets for SVD. Section 4 gives the outline of the existing, classical methods for SVD. Section 5 describes the state-of-the-art methods for SVD, and the paper is concluded in Section 6.

\section{Feature Extraction}

Singing voice detection is a crucial task that can be used to improve other tasks such as automatic lyrics alignment, singing melody transcription, vocal melody extraction, lyric transcription, singer identification, etc. To analyze music presence in a recorded audio signal, a representation that roughly corresponds to how people perceive sound through their auditory system has to be created. At a fundamental level, such audio representations aid in determining when events occur in time [23].

In order to locate vocal segments, researchers usually extract one or more types of features from the audio signals and then use a classifier to detect these audio features. The feature extraction stage is therefore critical for the subsequent classification process. Using a feature set (combining multiple features) usually results in better performance. Audio features provide the description of the sound that helps capture different aspects of sounds and build intelligent audio systems.

Audio features can be applied to feature extraction linked to audio effects [24], data classification [25], similarity measures [26], data mining [23], and feature-based synthesis [27], etc. Audio features can be categorized into three levels of abstraction: low-level such as spectral centroid, spectral flux, energy, zero-crossing rate; mid-level such as MFCCs; and high-level audio features such as lyrics, melody, and rhythm [28].

\section{Short-Time Fourier Transform Spectrum}

The Fourier transform is a mathematical formula for decomposing a signal into its individual frequencies and amplitudes. To put it another way, it converts the signal from the time domain to the frequency domain to create a spectrum. Perhaps short-time Fourier transform (STFT) spectrum is the most common time-frequency representation and has been widely used in various domains other than music processing. The STFT is also used 
to represent other audio features such as Mel-frequency cepstral coefficients (MFCCs) and chroma features [23].

Mel-spectrogram

The Mel-scale is a perceptual scale of pitches. A spectrogram is a visual image of a signal's frequency spectrum as it changes over time. A spectrogram is obtained by applying STFT on overlapping windowed segments of the signal. This spectrogram is a graphical way of representing STFT data. Mel-spectrogram is often used when applying deep learning approaches because it is more efficient than STFT spectrum [29].

\section{Temporal Features}

Temporal features describe a music signal's relatively long-term dynamics over time [30]. They are basically time-domain features, such as amplitude envelope, the energy of the signal, root mean square energy, zero-crossing rate (ZCR), etc., which are easy to extract. ZCR counts how many times the signal changes sign from negative to positive or vice versa in a specified time frame (in seconds). In the process of speech recognition and music information retrieval, ZCR is an essential feature in voice/noise classification. Speech can be unvoiced, and voiced fricatives (speech) have higher ZCR.

\section{Spectral features}

Spectral features, such as band energy ratio, spectral centroid, bandwidth, spectral roll-off, Mel-frequency cepstral coefficients (MFCC), perceptive linear prediction (PLP), linear prediction cepstral coefficients (LPCCs) [31], etc., are frequency domain features that are derived by converting the time domain into the frequency domain using the Fourier transform. The spectral features can be used to determine the rhythm, notes, pitch, and melody. Spectral centroid calculated as the weighted average of the frequencies in the signal is determined by a Fourier transformation with their magnitudes as weights. The spectral centroid is used to calculate a sound's brightness, and it is an important factor in describing musical timbre.

MFCCs are widely used in SVD [32] and were first introduced by Davis and Mermelstein in 1980 [33]. Kim et al. [34] compared MFCC and audio spectrum projection features, and they mentioned that MFCCs were better for feature extraction. The use of MFCCs has proven to be a powerful tool in music and voice recognition, and sound recognition in general. The MFCCs are calculated as follows:

$$
C(x(T))=F^{-1}[\log (F[x(t)])]
$$

where $x(t)$ is the time-domain signal. Figure 1 shows the steps to compute MFCC features. Calculation of the MFCC includes the following steps:

1. Division of the speech signals into frames, usually by applying a windowing function at fixed intervals [35];

2. Computing the coefficients of the discrete Fourier transform on each segment of windowed signal to convert the time domain into the frequency domain;

3. Taking the logarithm of the amplitude spectrum;

4. Smoothing the spectrum and emphasizing perceptually meaningful frequencies [35];

5. Taking the discrete cosine transform (DCT) of the list of mel log powers;

6. Generating cepstrum. 


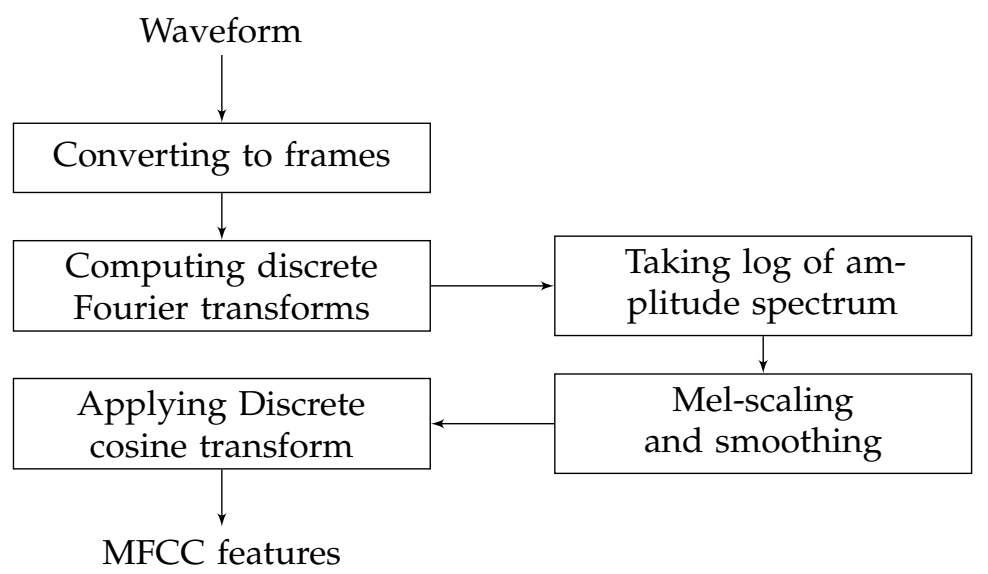

Figure 1. Calculation steps for MFCC features [35].

\section{Datasets}

This section outlines the most commonly used datasets to perform the SVD task. Most scientific papers related to the SVD task use small designed datasets such as Jamendo Corpus, RWC Popular Music, MedleyDB, MIR-1k, and iKala. This is due to the fact that it is better to have good quality reference datasets in order to achieve high accuracy in SVD tasks [36].

Table 1 describes the most commonly used datasets in the singing voice detection task and the related papers used for each dataset. Jamendo Corpus and RWC Popular Music datasets are the most popular for SVD.

Table 1. Public datasets relevant to singing voice detection.

\begin{tabular}{llll}
\hline Name & Number of Tracks & Size & Related Papers \\
\hline Jamendo Corpus & 93 & 443 mins & {$[16,17,21,37-41]$} \\
MedleyDB & 122 & 437 mins & {$[21,42]$} \\
MIR-1k & 1000 & 113 mins & {$[21,43]$} \\
RWC Popular Music & 100 & 407 mins & {$[17,21,37,40-42,44,45]$} \\
iKala & 352 & 176 mins & {$[21,42,46,47]$} \\
\hline
\end{tabular}

Jamendo Corpus is a public dataset consisting of 93 copyright-free songs. It was introduced by Mathieu Ramona et al. in [48]. It has vocal activation annotations. Moreover, for each song, the segments are annotated as "voice" or "no voice". The audio files in Jamendo are stereo. The dataset is divided into a training set containing 61 files, a validation set of 16 files and a test set of 16 files.

RWC Popular Music is a public dataset consisting of 100 pop songs with vocal activation and instrument annotations. It was introduced by Mauch et al. in [49]. It contains 80 Japanese songs and 20 English songs. The audio files in RWC are stereo and have a sampling frequency of $44.1 \mathrm{kHz}$ and 16 bits per sample.

MedleyDB is a multitrack dataset containing 122 tracks. It was introduced by Bitther et al. in [50]. It does not provide annotations for vocal or nonvocal segments, but it includes instrument activations, genre, and melody annotations. The audio format is WAV and has a sampling frequency of $44.1 \mathrm{kHz}$ with 16 bits per sample.

MIR-1k (Multimedia Information Retrieval lab) consists of 1000 songs with vocal activation and pitch contours annotations. MIR-1k was introduced by Chao-Ling Hsu et al. in [51]. For each song, the segments are annotated as "voice" and "no voice". The sampling rate is $16 \mathrm{kHz}$, and for all the 1000 clips, the clip duration is 4-13 s.

iKala dataset contains 352 30-seconds clips of Chinese popular songs. It was introduced by Chan et al. [52]. This dataset includes nonvocal regions and has a sampling frequency of $44.1 \mathrm{kHz}$. 


\section{Traditional Methods}

The singing voice detection task was first proposed by Berenzweig and Ellis in [13]. The authors focused on the problem of recognizing singing segments in popular music as a valuable and tractable method of music content analysis, and they used the statistical features and the hidden Markov model as a classifier. They were able to derive various statistics and models using Posterior Probability Features obtained from the acoustic classifier of a general-purpose speech recognizer. This approach enabled them to train an effective SVD system that was around $80 \%$ accurate at the frame level.

Namunu et al. [53] presented an approach for detecting singing voice boundaries derived from acoustical polyphonic music signals. They called this approach twice-iterated composite Fourier transform (TICFT). First, the music signal was divided into frames based on quarter notes. The harmonic structure of each frame was then measured using TICFT. Finally, the vocal and instrumental frames were classified using music domain knowledge. They mentioned that this method is less complex and more accurate than statistical learning methods. In terms of vocal boundary detection, they achieved over $80 \%$ frame-level accuracy.

Vembu et al. [54] presented a technique to identify vocal parts in music samples. They designed a classifier to perform a vocal-nonvocal segmentation task. They trained a neural network using several features: MFFCs, perceptual linear prediction (PLP), and log frequency power coefficients (LFPC), achieving the accuracy of $84.87 \%$ for the singing voice segmentation task. In [55], Lukashevich et al. used the autoregressive moving average model as a postprocessor reaching the accuracy of $82.5 \%$.

In [32], Rocamora and Herrera used various existing statistical descriptors and studied the accuracy of estimating vocal segments in music audio. They compare MFCCs, PLPs, LFPCs, and the harmonic coefficient (HC). The most appropriate feature was MFCC, and the best-performing classifier was the support vector machine. They also considered spectral features commonly used for instrument classification, such as centroid, roll-off, flux, skewness, kurtosis, and flatness. They reached the classification accuracy of $78.5 \%$ on the Jamendo dataset. In [15], Dittmar et al. suggested combining MFCCs with fluctogram variation and vocal variation. The authors used the random forest as a classifier and obtained the F-measure at the level of $87 \%$.

In [7], Li and Wang used a singing voice detection step before separating the vocals from the instrumental accompaniment. The authors used several features (MFCCs, linear prediction coefficients-LPC, PLP, and the $4-\mathrm{Hz}$ harmonic coefficient) and fed to a hidden Markov model (HMM) combined with the Viterbi algorithm [56]. Their 10-fold crossvalidation setup was based on only five rock and five country songs semiautomatically annotated from a karaoke $C D$. For training, several versions of each song with varying levels of signal-to-noise ratio (SNR) were generated, which was a type of data augmentation that has grown in popularity in recent years. The authors reported the accuracy of $80 \%, 85 \%$, $90 \%$, and $92 \%$, respectively, for $-5,0,5$, and $10 \mathrm{~dB}$ SNR.

Hsu et al. [51] proposed a singing voice separation system to identify and separate the unvoiced parts from the music accompaniment. The first stage of the system was singing voice detection. To decode music signals into the three groups-accompaniment, unvoiced, and voiced - the authors used hidden Markov models (HMMs). Then, they used Gaussian mixture models (GMMs) as states in a fully connected HMM and the Viterbi algorithm. In another work, Hsu et al. [43] used SVD for pitch estimation and vocal separation. They trained two GMMs to model vocal and nonvocal classes. The Viterbi algorithm was then used to decode the GMMs as states in a fully connected, continuous HMM.

In [48], Ramona et al. used several features such as MFCCs, ZCR, and sharpness. After a silent detection stage, these features were fed into a support vector machine as a classifier. On the output of the predicted sequence, the authors proposed a temporal smoothing strategy considering the temporal structure of the annotated segments. Instrumental portions less than 0.5 seconds in length were also smoothed out. On the publicly 
accessible Jamendo dataset, they reported accuracy of $82.2 \%$, as well as precise information on training and test set split.

In [38], Regnier et al. extracted sinusoidal partitions from musical audio signals and analyzed frequency modulation (vibrato) and amplitude modulation (tremolo) of each partition. They reached the accuracy of $76.8 \%$ by applying thresholds for vibrato and tremolo. A more advanced approach involving numerous characteristics and a GMM as a classifier result in a $77.4 \%$ for the F-measure.

Lehner et al. [39] proposed a real-time-capable and straightforward method to detect the presence of a human voice in audio signals. They used only MFCCs for feature representation and random forest as a classifier. They achieved an accuracy of $82.3 \%$ after the final optimization of the classifier parameters.

\section{Deep Learning Techniques}

In this section, we discuss the techniques used by researchers in the singing voice detection task with the help of deep learning techniques. Neural networks are widely used for solving this problem, and one of the recurrent neural network types, namely, the long short-term memory network, has been widely used by many researchers in SVD.

\subsection{Convolutional Neural Networks}

Convolutional neural networks (CNNs) are similar to traditional artificial neural networks (ANNs) in that they are made up of neurons that optimize themselves through learning. Each neuron continues to receive input and perform an operation (such as a scalar product followed by a nonlinear function), which is the foundation of many ANNs [57].

Schlüter et al. [17] introduced a model for singing voice detection using CNN. They used three-by-three 2D convolution layers. The model is capable of learning invariance by data augmentation. In the training phase, the authors applied data augmentation, such as time stretching and pitch shifting, on the audio representation. They developed several augmentation methods that can be efficiently used to work on spectrograms or Mel-spectrograms. Two of the augmentation techniques are data independent, while four are audio data specific, and one is specific to binary sequence labeling.

You et al. [18] applied the CNN model for singing voice detection with MFCC features, fast Fourier transform (FFT) features, raw pulse-code modulation (PCM) samples, and long short-term memory. They called it CNN for MFCC feature (MCNN), CNN for spectrogram (SCNN), end-to-end CNN for raw PCM samples (ECNN), and convolutional LSTM (CLSTM). MCNN, SCNN, CLSTM, and ECNN were trained and tested using the Jamendo Corpus dataset and achieved the accuracy of $88.2 \%, 91.8 \%, 77.1 \%$, and 90.4\%, respectively. SCNN achieved the best accuracy after ten trials on the Jamendo dataset.

Huang et al. [58] proposed various structures of CNN for SVD. The input features were MFCC, discrete Fourier transform (DFT) coefficients, and raw PCM samples. The authors found out that DFT coefficients achieved higher detection accuracy (up to 92\%) evaluated on all epochs over the average of 10 trials which is higher than MFCC and raw PCM.

In [59], Wenming Gui et al. have significantly improved CNN presented in [29] by adding batch normalization, changing the activation function to Leaky ReLU, and analyzing attention distribution of the feature maps. The numerical results were achieved on Jamendo Corpus, RWC Popular Music, and MIR-1k datasets.

Krause et al. in [60] analyzed the generalization capabilities and robustness of two models in a different scenario. They used opera recordings as a dataset. The studies were performed for one standard classifier-random forest-and one based on deep learning technique-CNN [15]. The quantitative results have shown that CNN outcomes are slightly better than those obtained with the random forest classifier. 


\subsection{Recurrent Neural Networks}

A recurrent neural network (RNN) is a computational neural network with feedback connections. RNNs can deal with time-series signals such as audio and video effectively and flexibly [61]. In a simple RNN, the hidden state at a time $t$ is computed as follows:

$$
\begin{gathered}
h_{t}=f\left(W_{i h} i_{t}+W_{h h} h_{t-1}+b_{h}\right) \\
z_{t}=f\left(W_{h z} h_{t}+b_{z}\right)
\end{gathered}
$$

where $f$ is an activation function; $h_{t} \in \mathbb{R}$ is the hidden state with $N$ hidden units; $W_{i h}$ represents weight matrices of connections between input and hidden layers; $i_{t}$ is the input at time $t ; b$ denotes the bias vector; $z_{t}$ is an output vector; and $W_{h h}$ represents weight matrices of connections between hidden and hidden layers.

RNNs are used to process sequential data in such a way that each data point can be understood in a context. RNNs have demonstrated success in tasks such as text generation [62] and speech recognition [63]. They can be used to model nonlinear sequential relationships, but it is hard to train a simple RNN due to vanishing gradient and exploding problems, and the problem of long-term dependencies.

Hughes et al. [64] proposed a recurrent neural network model for voice activity detection. The model is multilayered, where the nodes compute quadratic polynomials, and all proposed model parameters are optimized together. The authors have shown that the proposed model can outperform larger GMM-based systems on voice activity detection tasks.

\subsection{Long Short-Term Memory}

Long Short-Term Memory (LSTM) is a special kind of RNN that can learn long-term dependencies. Moreover, it is designed to avoid the long-term dependency problem. Remembering information for extended periods is practically LSTM's default behavior [65].

As presented in Figure 2, each LSTM block includes a memory cell. The input and forget gates monitor the content of the network while it is performing classification at each time level. The input of the block to which it belongs can be stored in the cell for as long as it is required. LSTM cell can be described as follows:

- Forget gate: $f_{t}=\sigma\left(W_{f} \cdot\left[h_{t-1}, x_{t}\right]+b_{f}\right)$

- Input gate: $i_{t}=\sigma\left(W_{i} \cdot\left[h_{t-1}, x_{t}\right]+b_{i}\right)$

$$
\tilde{C}_{t}=\tanh \left(W_{c} \cdot\left[h_{t-1}, x_{t}\right]+b_{C}\right)
$$

- $\quad$ Cell state: $C_{t}=f_{t} * C_{t_{1}}+i_{t} * \tilde{C}_{t}$

- Output gate: $i_{t}=\sigma\left(W_{0} \cdot\left[h_{t-1}, x_{t}\right]+b_{o}\right)$

$$
h_{t}=o_{t} * \tanh \left(C_{t}\right)
$$

where "." is the element-wise product, $i_{t}$ represents the input gate, $f_{t}$ represents the forget gate, $o_{t}$ represents the output gate, $\sigma$ represents the sigmoid function, $w_{x}$ is the weight for the respective gate $(x), h_{t-1}$ is the output for the previous LSTM block at $(t-1)$ timestamp, $x_{t}$ is an input at current timestamp, and $b_{x}$ are biases for the respective gates $(x)$.

A typical LSTM cell has three gates: a forget gate, an input gate, and an output gate. The forget gate of the LSTM cell determines how much of the previous data should be forgotten. The input gate determines how much information is written to the internal cell state. The output gate determines the next hidden state to be generated from the current internal cell state. LSTM units have a single memory cell that allows them to store data for an indefinite period. This memory cell's read, write, and delete operations are handled by gates that function similarly to standard units.

Not only are the hidden units connected to the input units (or, in the case of consecutive hidden layers, to the units of the preceding hidden layer), but each unit is also connected to itself, i.e., the previous time step. The RNN has access to past information via these recurrent connections to model temporal context. 


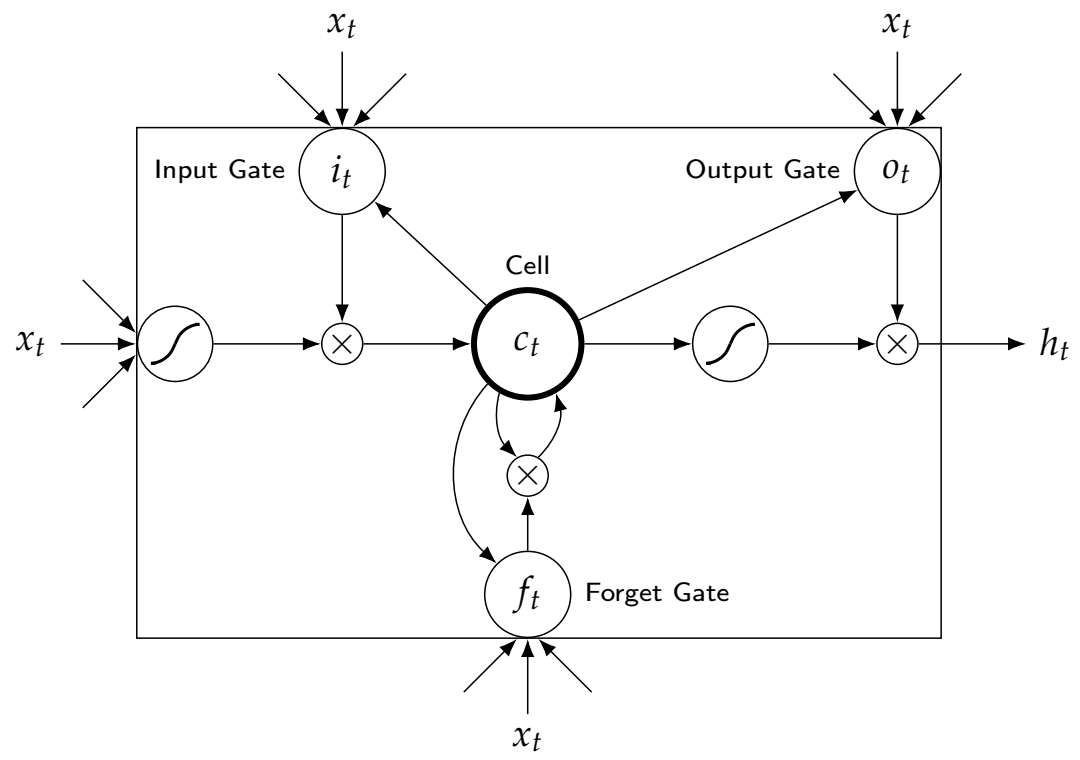

Figure 2. LSTM cell.

Using LSTM-RNN seems to be one of the best choices for singing voice detection. Eyben et al. [66] presented a data-driven method to voice activity detection trained on RASTA-PLP as front-end features based on long short-term memory-recurrent neural network (LSTM-RNN). The results show that LSTM-RNN outperforms all other methods in statistical benchmarks.

Lehner et al. [37] used LSTM-RNN for singing voice detection. They applied a unidirectional RNN with one hidden layer and 55 LSTMs to sort out frames into vocal and nonvocal. The input was based on several audio features in the feature representation that included 30 MFCCs. The authors achieved state-of-the-art performance on Jamendo and RWC datasets.

\subsection{Bidirectional LSTMs}

Bidirectional RNN (BRNN) are simply two separate RNNs joined together. The idea behind BRNN is to divide the state neurons of a regular RNN into two parts: one for the positive time direction (forward states) and one for the negative time direction (backward states). RNNs can only use a past temporal context. When the entire sequence of input features is available, it can also be possible to take advantage of the future context. This can be accomplished with a bidirectional RNN (BRNN). When it comes to learning long-term dependencies, LSTM-RNNs have proved to be superior to regular RNNs [67].

Leglaive et al. [16] combined deep BRNNs and LSTM to form deep BLSTM-RNNs and make use of a long-range past and future temporal context in order to classify each input vector. Figure 3 illustrates the system used for this experiment. A system has two-stage harmonic-percussion source separation (HPSS) [68] for the classifier input to extract signals specific to the singing voice. Mel-spectrograms of the obtained harmonic and percussive components are combined as an input for the classifier for each frame. The output predictions for each input frame are produced by several recurrent layers followed by a shared densely connected layer. This classifier can use the inherent sequential aspect of short-term feature extraction in a piece of music to decide on the presence/absence of a singing voice in the past and future temporal context. The authors compared BLSTM with a support vector machine for singing voice segmentation and achieved the accuracy of $91.5 \%$ for BLSTM on the Jamendo dataset outperforming other approaches. 


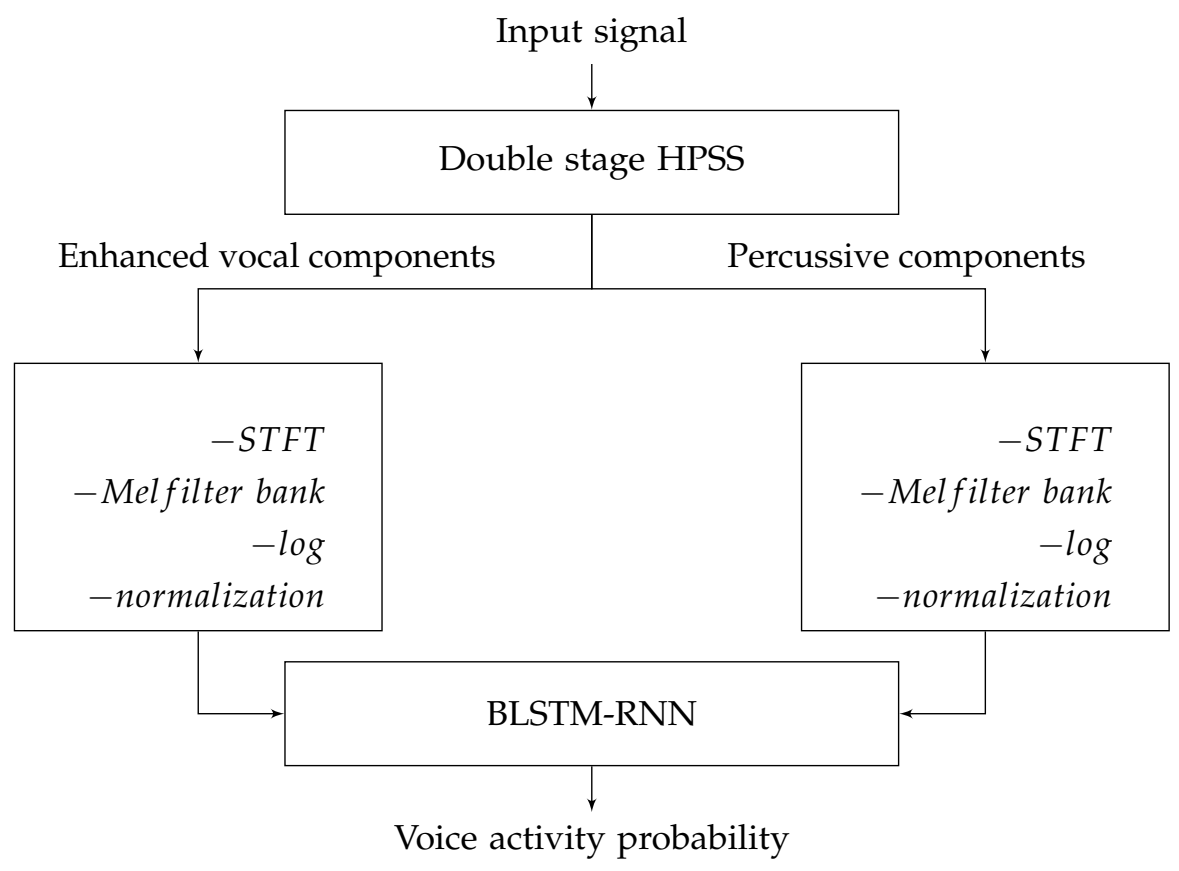

Figure 3. The overview of the BLSTM architecture used for SVD in [16].

\subsection{GRU-RNN}

Cho et al. suggested a gated recurrent unit (GRU) [69] to allow each recurrent unit to capture dependencies across time scales adaptively. The GRU, like the LSTM, has gating units that modulate the flow of information within the unit but without the need for separate memory cells. There are a few differences between GRU and LSTM. GRU reveals its entire content without any monitoring, while LSTM manages the memory content's exposure-in other words, GRU has a more straightforward structure than LSTM. Another distinction is the addition of new memory content to the system. The update gate is used to monitor information flow in GRU, while the forget gate is used independently in LSTM.

Chen et al. [41] proposed a system (Figure 4) based on GRU-RNN. The preprocessing step used Deep U-Net convolutional networks for singing voice separation. Then, the authors extracted features and fed them to the classifier. The extracted features were MFCC, Mel-filter bank, LPCC, and chroma features. They showed a unidirectional RNN with a hidden layer of 60 GRU units. The classifier's output is either 1 or 0 , with 1 indicating singing and 0 indicating nonsinging. The authors set the block duration as 120 and $720 \mathrm{~ms}$ with the temporal smoothing postprocessing step. The authors applied this system for Jamendo and RWC Popular Music datasets. The results are shown in Tables 2 and 3 for the GRU-RNN (2) with a block duration of $120 \mathrm{~ms}$ and GRU-RNN (3) with a block duration of $720 \mathrm{~ms}$.

\subsection{ConvLSTM or $L R C N$}

Convolutional LSTM (ConvLSTM) networks or long short-term recurrent convolutional networks (LRCNs) have a wide range of applications, including video classification, image captioning, image classification, activity recognition, image labeling, video captioning, singing voice detection, etc. LRCNs can capture both features of CNNs and RNNs by combining them from spatial and temporal features. The LRCN was first proposed in [70]. ConvLSTM is a type of RNN for a spatiotemporal prediction that employs convolutional structures in both input-to-state and state-to-state transitions. 


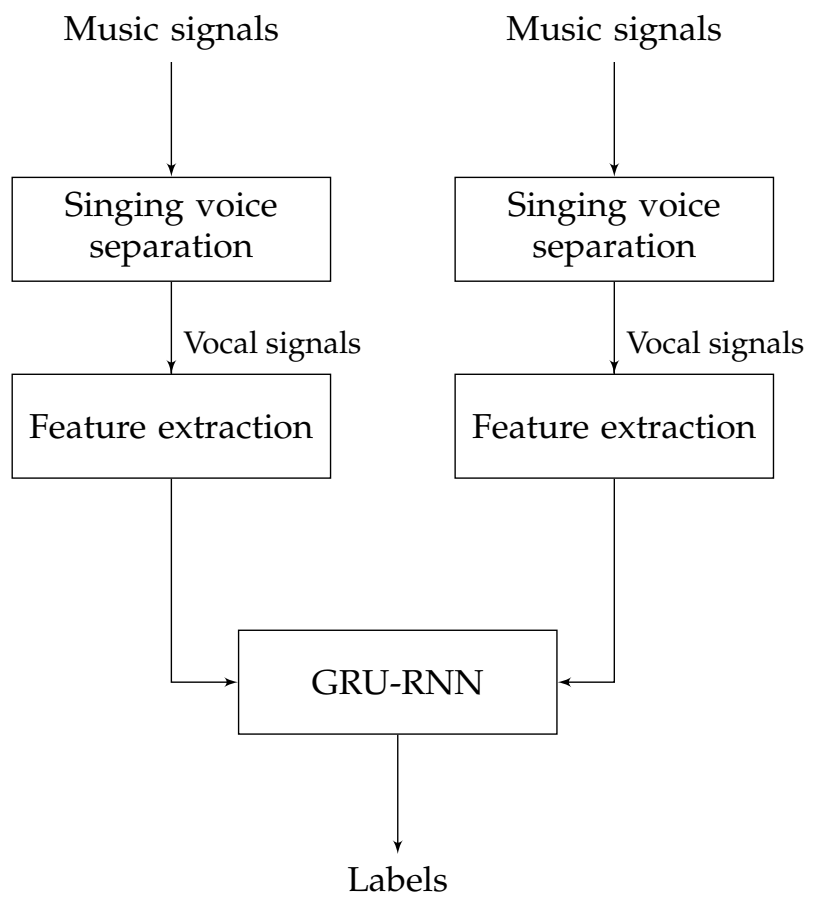

Figure 4. The overview of the GRU-RNN architecture used in [41].

Table 2. Comparison of existing singing voice detection methods on the Jamendo Corpus dataset.

\begin{tabular}{lrrrrrr}
\hline & & Year & \multicolumn{3}{c}{ Evaluation Measures (in [\%]) } \\
\cline { 6 - 7 } Method & Author & Published & Accuracy & Precision & Recall & F-Measure \\
\hline SVM & Ramona [48] & 2008 & 82.2 & - & - & 84.3 \\
GMM & Regnier et al. [38] & 2009 & - & - & - & 77 \\
Random forest & Lehner et al. [39] & 2013 & 84.8 & - & - & 84.6 \\
Feature Engineering & Lehner et al. [71] & 2014 & 88.2 & 88 & 86.2 & 87.1 \\
LSTM-RNN (1) & Lehner et al. [37] & 2015 & 91.5 & 89.8 & 90.6 & 90.2 \\
LSTM-RNN (2) & Zhang et al. [21] & 2020 & 89.5 & 89.5 & 89.6 & 88.8 \\
CNN (1) & Schlüter et al. [17] & 2015 & 92.3 & - & 90.3 & - \\
CNN (2) & Zhang et al. [21] & 2020 & 90.4 & 90.6 & 90.4 & 90.3 \\
CNN (3) & Gui et al. [59] & 2021 & 88.9 & 91.4 & 89.9 & 90.6 \\
Bi-LSTMs & Leglaive et al. [16] & 2015 & 91.5 & 89.5 & 92.6 & 91 \\
Bootstrapping procedure & Dittmar et al. [15] & 2015 & 88.2 & - & - & 87 \\
GRU-RNN (1) & Zhang et al. [21] & 2020 & 91 & 90.8 & 91.2 & 91.4 \\
GRU-RNN (2) & Chen et al. [41] & 2019 & 88.2 & 85.39 & 92.78 & 88.93 \\
GRU-RNN (3) & Chen et al. [41] & 2019 & 90.8 & 98.2 & 93.3 & 91.2 \\
LRCN & Zhang et al. [21] & 2020 & 91.6 & 92.6 & 93.4 & 93 \\
\hline
\end{tabular}


Table 3. Comparison of existing singing voice detection methods on the RWC Popular Music dataset.

\begin{tabular}{lrrrrrr}
\hline & & Year & \multicolumn{3}{c}{ Evaluation Measures(in [\%]) } \\
\cline { 5 - 7 } Method & Author & Published & Accuracy & Precision & Recall & F-Measure \\
\hline SVM-HMM & Mauch [45] & 2011 & 87.2 & 88.7 & 92.1 & 90.4 \\
Random forest & Lehner et al. [39] & 2013 & 86.8 & 87.9 & 90.6 & 89.2 \\
Feature Engineering & Lehner et al. [71] & 2014 & 87.5 & 87.5 & 92.6 & 90 \\
LSTM-RNN (1) & Lehner et al. [37] & 2015 & 92.3 & 93.8 & 93.4 & 93.6 \\
LSTM-RNN (2) & X. Zhang et al. [21] & 2020 & 93.7 & 94.1 & 93.3 & 92.8 \\
CNN (1) & Schlüter et al. [16] & 2015 & 92.7 & - & 93.5 & - \\
CNN (2) & X. Zhang et al. [21] & 2020 & 94 & 93.6 & 94 & 94.2 \\
CNN (3) & Gui et al. [59] & 2021 & 88.9 & 90.7 & 97.0 & 93.7 \\
GRU-RNN (1) & X. Zhang et al. [21] & 2020 & 95.2 & 95.1 & 95.3 & 95.3 \\
GRU-RNN (2) & Chen et al. [41] & 2019 & 92.1 & 92.7 & 95.4 & 94 \\
GRU-RNN (3) & Chen et al. [41] & 2019 & 95.3 & 96.1 & 96.9 & 96.5 \\
LRCN & X. Zhang et al. [21] & 2020 & 97 & 97.1 & 96.8 & 96.3 \\
\hline
\end{tabular}

You et al. [18] proposed a convolutional LSTM for singing voice detection. The authors mentioned that CLSTM (convolutional LSTM) might theoretically outperform the typical LSTM network with spectrogram inputs because it uses numerous two-dimensional planes as inputs. Figure 5 illustrates the CLSTM network that uses three subplanes as the input sequence.

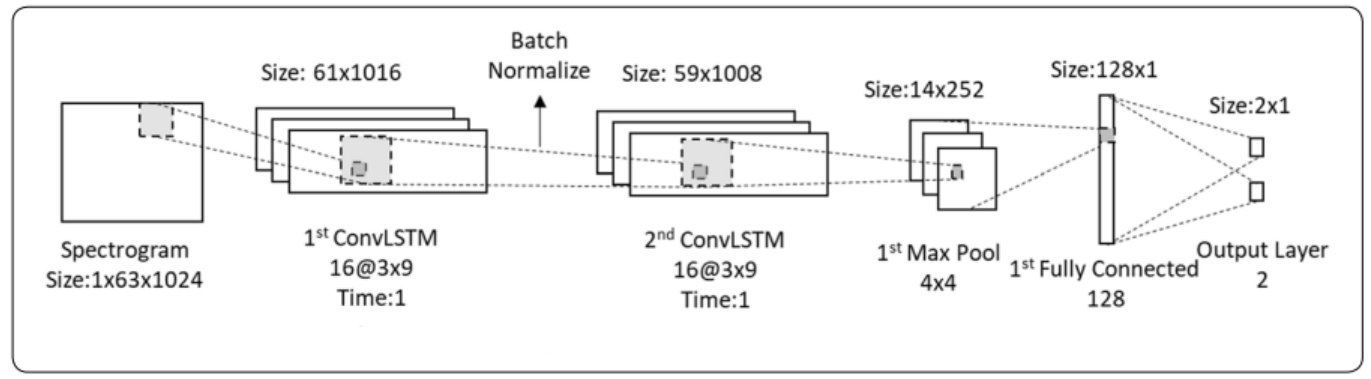

Figure 5. Convolutional LSTM used in [18] (used under the terms and conditions of the Creative Commons Attribution (CC BY) license (http:/ / creativecommons.org/licenses/by/4.0/) (accessed on 10 November 2021)).

Zhang et al. [21] proposed LRCN to extract the crucial features that represent the audio content in the frequency domain and characterize the vocal background in the time domain for vocal detection. As presented in Figure 6, the network for detecting singing voices has an input layer that is the same size as the combined acoustic feature vector, three hidden layers, and an output layer with a single sigmoid unit. The network has been trained as a classifier to output vocal scores in a value space of 0 and 1 for each frameblock, where 1 represents a singing voice and 0 represents no singing part of the song. The LSTM layer in LRCN learns the temporal relationship from the features encoded by the convolutional layer. By contrast, the convolutional layer spatially adopts the combined audio features for deep feature extraction. In [21], the proposed system's architecture employs singing voice separation as a preprocessing technique for obtaining vocal signals. It is then accompanied by a standard classification method, in which the authors applied machine learning techniques (the $\mathrm{LRCN}$ ) to successive frames of input vocal signals with a collection of audio features. The authors proposed the LRCN model on five different datasets (RWC, Jamendo, MIR-1K, iKala, and MedleyDB), which were mentioned before in Section 3. On the Jamendo dataset, they reached the accuracy of $92 \%$ and 0.93 for the 
f1-score; on the RWC dataset-the accuracy of $97 \%$ and 0.96 for the f1-score; on MIR-1K dataset-the accuracy of $94 \%$ and 0.89 for the f1-score; on the iKala dataset-the accuracy of $99 \%$ and 0.99 for the f1-score; and on the MedleyDB-the accuracy of $81 \%$ and 0.79 for the f1-score. They also compared the proposed LRCN model with the existing methods for singing voice detection on the Jamendo Corpus dataset and RWC Popular Music datasets. The results show that LRCN exhibited a state-of-the-art performance.

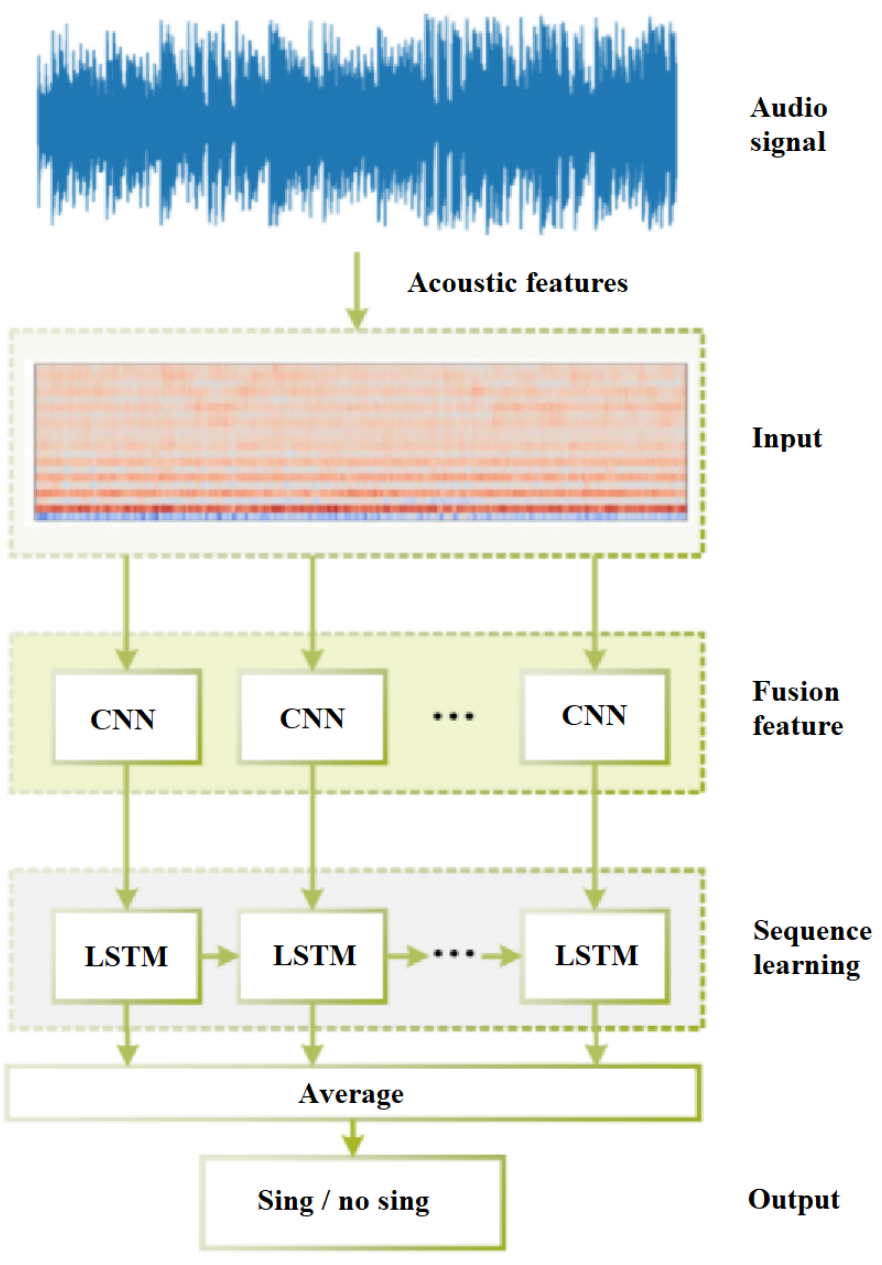

Figure 6. The topology of L RCN used in [21] (used under the terms and conditions of the Creative Commons Attribution (CC BY) license (http:/ / creativecommons.org/licenses/by/4.0/) (accessed on 10 November 2021)).

Figure 7 illustrates the structure of the LRCN layer. The key equations of LRCN are as follows:

- Input gate: $i(t)=\sigma\left(W_{i} \cdot[\operatorname{Conv}(X(t)), H(t-1), C(t-1)]+b_{i}\right)$

- forget gate: $f(t)=\sigma\left(W_{f} \cdot[\operatorname{Conv}(X(t)), H(t-1)+C(t-1)]+b_{f}\right)$

- $\quad$ LRCN Cell: $C(t)=f(t) * C(t-1)+i(t) \cdot \tanh \left(W_{c} \cdot[\operatorname{Conv}(X(t)), H(t-1)]+b_{c}\right)$

- Output gate: $o_{t}=\sigma\left(W_{0} \cdot[\operatorname{Conv}(X(t)), H(t-1), C(t)]+b_{0}\right)$

- Hidden state: $H(t)=o(t) \cdot \tanh (C(t))$

where "." is the element-wise product, conv is the convolution operator, $i_{t}$ represents input gate, $\sigma$ represents sigmoid function, $w_{x}$ is the weight matrix for the respective gate $(x), h_{t-1}$ is the output for the previous LSTM block at $(t-1)$ timestamp, $x_{t}$ is an input at current timestamp, and $b_{x}$ are biases for the respective gates $(x)$. 


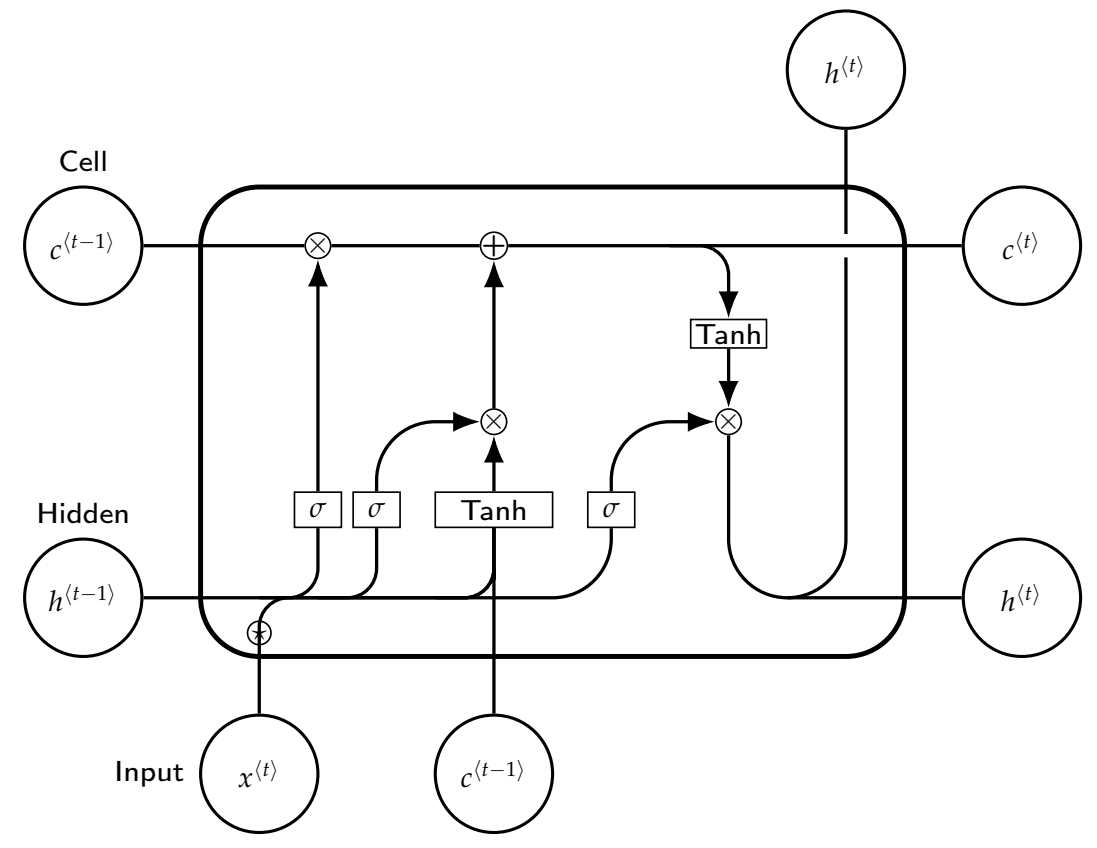

Figure 7. Inner structure of LRCN layer used in [21] (used under the terms and conditions of the Creative Commons Attribution (CC BY) license (http:/ / creativecommons.org/licenses/by/4.0/) (accessed on 10 November 2021)).

Tables 2 and 3 summarize the existing SVD methods applied on the Jamendo Corpus and RWC Popular Music, respectively. Accuracy is the proportion of correctly classified frames. The recall is the estimated proportion of frames labeled as voiced in the ground truth. Precision is the percentage of frames that are effectively voiced in the ground truth that is measured as voiced by the algorithm. F-measure (also called F1-score) combines the precision and recall of the model and is used to measure the accuracy of the model on a dataset. The number of false-negative (FN), true-negative (TN), false-positive (FP), and true-positive (TP) results accumulated across all songs in the testing set was calculated by comparing model predictions to ground-truth labels. The four evaluation metrics can be represented as follows:

$$
\begin{gathered}
\text { Recall }=\frac{T P}{T P+F N^{\prime}}, \\
\text { Precision }=\frac{T P}{T P+F P^{\prime}} \\
\text { Accuracy }=\frac{T P+T N}{\text { totalframes }}, \\
F_{1}=2 * \frac{\text { precision } * \text { recall }}{\text { precision }+ \text { recall }} .
\end{gathered}
$$

The comparison of the SVD methods in Tables 2 and 3 shows that LRCN and GRU achieved the best results in terms of accuracy, precision, recall, and F-measure.

\section{Conclusions and Discussion}

This paper presents a survey on existing singing voice detection methods. Many possible features can be used for SVD. The long-term recurrent convolutional network achieved state-of-the-art results on both Jamendo Corpus and RWC Popular Music datasets. GRU has a more straightforward structure and higher computational efficiency than LSTM; therefore, GRU achieved better accuracy in the singing voice detection task. We can notice that, by using any algorithm on the Jamendo and RWC, the accuracy on the RWC dataset is always higher than on the Jamendo. 
RNNs are good at processing sequence data and making predictions, but they have short-term memory problems. LSTMs and GRUs were developed as a way to reduce short-term memory by using gate mechanisms. Gates are basically neural networks that govern data flow through the sequence chain.

We believe that future works in this field will be focused on the development of bidirectional LSTM, ConvLSTM, and GRU-RNN on DALI [36], Jamendo, and RWC datasets. Researchers will likely turn to knowledge distillation and attention-based mechanisms due to their growing popularity $[59,72]$.

To the best of our knowledge, no recent review article has been written on the singing voice detection problem. As a result, we hope that this research paper can be significant for future research in that it gives a deep understanding of the models and techniques used in SVD.

Author Contributions: Conceptualization, D.K. and R.M.; methodology, D.K. and D.M.; writingoriginal draft preparation, R.M. and D.K.; writing-review and editing, D.K., D.M. and R.M.; visualization, R.M.; supervision, D.M. and D.K.; project administration, D.K.; funding acquisition, D.K. and D.M. All authors have read and agreed to the published version of the manuscript.

Funding: This work was partially supported by Statutory Research funds of the Department of Applied Informatics, Silesian University of Technology, Gliwice, Poland, and partially, by the professorship grant (02/020/RGP19/0184) and the pro-quality grant for highly scored publications or issued patents of the Rector of the Silesian University of Technology, Gliwice, Poland (grant No 02/100/RGJ21/0009).

Institutional Review Board Statement: Not applicable.

Informed Consent Statement: Not applicable.

Data Availability Statement: The data is contained within the article.

Conflicts of Interest: The authors declare no conflict of interest.

\section{References}

1. Wong, C.H.; Szeto, W.M.; Wong, K.H. Automatic lyrics alignment for Cantonese popular music. Multimed. Syst. 2007, 12, 307-323. [CrossRef]

2. Fujihara, H.; Goto, M. Lyrics-to-audio alignment and its application. In Dagstuhl Follow-Ups; Schloss Dagstuhl-Leibniz-Zentrum fuer Informatik: Wadern, Germany, 2012; Volume 3. [CrossRef]

3. Kan, M.Y.; Wang, Y.; Iskandar, D.; Nwe, T.L.; Shenoy, A. LyricAlly: Automatic synchronization of textual lyrics to acoustic music signals. IEEE Trans. Audio Speech Lang. Process. 2008, 16, 338-349. [CrossRef]

4. Rigaud, F.; Radenen, M. Singing Voice Melody Transcription Using Deep Neural Networks. In Proceedings of the 17th ISMIR Conference, New York, NY, USA, 7-11 August 2016; pp. 737-743.

5. Bittner, R.M.; McFee, B.; Salamon, J.; Li, P.; Bello, J.P. Deep Salience Representations for F0 Estimation in Polyphonic Music. In Proceedings of the 18th International Society for Music Information Retrieval Conference (ISMIR 2017), Suzhou, China, 23-27 October 2017; pp. 63-70.

6. Pardo, B.; Rafii, Z.; Duan, Z. Audio source separation in a musical context. In Springer Handbook of Systematic Musicology; Springer: Berlin/Heidelberg, Germany, 2018; pp. 285-298.

7. Li, Y.; Wang, D. Separation of singing voice from music accompaniment for monaural recordings. IEEE Trans. Audio Speech Lang. Process. 2007, 15, 1475-1487. [CrossRef]

8. Jansson, A.; Humphrey, E.; Montecchio, N.; Bittner, R.; Kumar, A.; Weyde, T. Singing voice separation with deep u-net convolutional networks. In Proceedings of the 18th International Society for Music Information Retrieval Conference, Suzhou, China, 23-27 October 2017.

9. Rao, V.; Rao, P. Vocal melody extraction in the presence of pitched accompaniment in polyphonic music. IEEE Trans. Audio Speech Lang. Process. 2010, 18, 2145-2154. [CrossRef]

10. Hosoya, T.; Suzuki, M.; Ito, A.; Makino, S.; Smith, L.A.; Bainbridge, D.; Witten, I.H. Lyrics Recognition from a Singing Voice Based on Finite State Automaton for Music Information Retrieval. In Proceedings of the 6th International Conference on Music Information Retrieval (ISMIR 2005), London, UK, 11-15 September 2005; pp. 532-535.

11. McVicar, M.; Ellis, D.P.; Goto, M. Leveraging repetition for improved automatic lyric transcription in popular music. In Proceedings of the 2014 IEEE International Conference on Acoustics, Speech and Signal Processing (ICASSP), Florence, Italy, 4-9 May 2014; pp. 3117-3121. [CrossRef] 
12. Zhang, T. Automatic singer identification. In Proceedings of the 2003 International Conference on Multimedia and Expo. ICME'03. Proceedings (Cat. No. 03TH8698), Baltimore, MD, USA, 6-9 July 2003.

13. Berenzweig, A.L.; Ellis, D.P. Locating singing voice segments within music signals. In Proceedings of the 2001 IEEE Workshop on the Applications of Signal Processing to Audio and Acoustics (Cat. No. 01TH8575), New Platz, NY, USA, 24-24 October 2001; pp. 119-122. doi: 10.1109/ASPAA.2001.969557 [CrossRef]

14. Kim, Y.E.; Whitman, B. Singer identification in popular music recordings using voice coding features. In Proceedings of the 3rd International Conference on Music Information Retrieval, Paris, France, 13-17 October 2002; Volume 13, p. 17.

15. Dittmar, C.; Lehner, B.; Prätzlich, T.; Müller, M.; Widmer, G. Cross-Version Singing Voice Detection in Classical Opera Recordings In Proceedings of the International Conference on Music Information Retrieval (ISMIR), Malaga, Spain, 26-30 October 2015; pp. 618-624.

16. Leglaive, S.; Hennequin, R.; Badeau, R. Singing voice detection with deep recurrent neural networks. In Proceedings of the 2015 IEEE International Conference on Acoustics, Speech and Signal Processing (ICASSP), South Brisbane, QLD, Australia, 19-24 April 2015; pp. 121-125.

17. Schlüter, J.; Grill, T. Exploring Data Augmentation for Improved Singing Voice Detection with Neural Networks. In Proceedings of the 16th International Society for Music Information Retrieval Conference (ISMIR 2015), Malaga, Spain, 26-30 October 2015; pp. 121-126.

18. You, S.D.; Liu, C.H.; Chen, W.K. Comparative study of singing voice detection based on deep neural networks and ensemble learning. Hum.-Centric Comput. Inf. Sci. 2018, 8, 34. [CrossRef]

19. Ohishi, Y.; Goto, M.; Itou, K.; Takeda, K. Discrimination between singing and speaking voices. In Proceedings of the Ninth European Conference on Speech Communication and Technology, Lisboa, Portugal, 4-8 September 2005.

20. Vijayan, K.; Li, H.; Toda, T. Speech-to-singing voice conversion: The challenges and strategies for improving vocal conversion processes. IEEE Signal Process. Mag. 2018, 36, 95-102. [CrossRef]

21. Zhang, X.; Yu, Y.; Gao, Y.; Chen, X.; Li, W. Research on Singing Voice Detection Based on a Long-Term Recurrent Convolutional Network with Vocal Separation and Temporal Smoothing. Electronics 2020, 9, 1458. [CrossRef]

22. Rani, B.; Rani, A.J.; Ravi, T.; Sree, M.D. Basic fundamental recognition of voiced, unvoiced, and silence region of a speech. Int. J. Eng. Adv. Technol. 2014, 4, 83-86

23. Li, T.; Ogihara, M.; Tzanetakis, G. Music Data Mining; CRC Press: Boca Raton, FL, USA, 2011.

24. Stables, R.; Enderby, S.; De Man, B.; Fazekas, G.; Reiss, J.D. Safe: A System for Extraction and Retrieval of Semantic Audio Descriptors. In Electronic Engineering and Computer Science; Queen Mary University of London: London, UK, 2014.

25. McKinney, M.; Breebaart, J. Features for audio and music classification. In Proceedings of the ISMIR2003, Baltimore, MD, USA, 27-30 October 2003.

26. Gygi, B.; Kidd, G.R.; Watson, C.S. Similarity and categorization of environmental sounds. Percept. Psychophys. 2007, 69, 839-855. [CrossRef]

27. Hoffman, M.D.; Cook, P.R. Feature-Based Synthesis: A Tool for Evaluating, Designing, and Interacting with Music IR Systems. In Proceedings of the ISMIR 2006, 7th International Conference on Music Information Retrieval, Victoria, BC, Canada, 8-12 October 2006; pp. 361-362.

28. Knees, P.; Schedl, M. Music Similarity and Retrieval: An Introduction to Audio-and Web-Based Strategies; The Information Retrieval Series; Springer: Berlin/Heidelberg, Germany, 2016.

29. Lee, K.; Choi, K.; Nam, J. Revisiting Singing Voice Detection: A quantitative review and the future outlook. In Proceedings of the 19th International Society for Music Information Retrieval Conference, ISMIR 2018, Paris, France, 23-27 September 2018; pp. 506-513.

30. Jeong, I.Y.; Lee, K. Learning Temporal Features Using a Deep Neural Network and its Application to Music Genre Classification. In Proceedings of the 17th International Society for Music Information Retrieval Conference (ISMIR), New York, NY, USA, 7-11 August 2016; pp. 434-440.

31. Gupta, H.; Gupta, D. LPC and LPCC method of feature extraction in Speech Recognition System. In Proceedings of the 2016 6th International Conference-Cloud System and Big Data Engineering (Confluence), Noida, India, 14-15 January 2016; pp. 498-502. [CrossRef]

32. Rocamora, M.; Herrera, P. Comparing audio descriptors for singing voice detection in music audio files. In Proceedings of the Brazilian Symposium on Computer Music, 11th, São Paulo, Brazil, 1-3 September 2007; Volume 26, p. 27.

33. Davis, S.; Mermelstein, P. Comparison of parametric representations for monosyllabic word recognition in continuously spoken sentences. IEEE Trans. Acoust. Speech Signal Process. 1980, 28, 357-366. [CrossRef]

34. Kim, H.G.; Sikora, T. Comparison of MPEG-7 audio spectrum projection features and MFCC applied to speaker recognition, sound classification and audio segmentation. In Proceedings of the 2004 IEEE International Conference on Acoustics, Speech, and Signal Processing, Montreal, QC, Canada, 17-21 May 2004; Volume 5.

35. Logan, B. Mel frequency cepstral coefficients for music modeling. In Proceedings of the International Symposium on Music Information Retrieval, Plymouth, MA, USA, 23-25 October 2000.

36. Meseguer-Brocal, G.; Cohen-Hadria, A.; Peeters, G. Dali: A large dataset of synchronized audio, lyrics and notes, automatically created using teacher-student machine learning paradigm. arXiv 2019, arXiv:1906.10606. 
37. Lehner, B.; Widmer, G.; Bock, S. A low-latency, real-time-capable singing voice detection method with LSTM recurrent neural networks. In Proceedings of the 2015 23rd European Signal Processing Conference (EUSIPCO), Nice, France, 31 August-4 September 2015; pp. 21-25.

38. Regnier, L.; Peeters, G. Singing voice detection in music tracks using direct voice vibrato detection. In Proceedings of the 2009 IEEE International Conference on Acoustics, Speech and Signal Processing, Taipei, Taiwan, 19-24 April 2009; pp. 1685-1688.

39. Lehner, B.; Sonnleitner, R.; Widmer, G. Towards Light-Weight, Real-Time-Capable Singing Voice Detection. In Proceedings of the 14th International Conference on Music Information Retrieval (ISMIR 2013), Curitiba, Brazil, 4-8 November 2013.

40. Schlüter, J. Learning to Pinpoint Singing Voice from Weakly Labeled Examples. In Proceedings of the 17th ISMIR Conference, New York, NY, USA, 7-11 August 2016; pp. 44-50.

41. Chen, Z.; Zhang, X.; Deng, J.; Li, J.; Jiang, Y.; Li, W. A Practical Singing Voice Detection System Based on GRU-RNN. In Proceedings of the 6th Conference on Sound and Music Technology (CSMT); Springer: Singapore, 2019; pp. 15-25._2 [CrossRef]

42. Kum, S.; Nam, J. Joint detection and classification of singing voice melody using convolutional recurrent neural networks. Appl. Sci. 2019, 9, 1324. [CrossRef]

43. Hsu, C.L.; Wang, D.; Jang, J.S.R.; Hu, K. A tandem algorithm for singing pitch extraction and voice separation from music accompaniment. IEEE Trans. Audio Speech Lang. Process. 2012, 20, 1482-1491. [CrossRef]

44. Song, L.; Li, M.; Yan, Y. Automatic Vocal Segments Detection in Popular Music. In Proceedings of the 2013 Ninth International Conference on Computational Intelligence and Security, Emeishan, China, 14-15 December 2013; pp. 349-352. [CrossRef]

45. Mauch, M.; Fujihara, H.; Yoshii, K.; Goto, M. Timbre and Melody Features for the Recognition of Vocal Activity and Instrumental Solos in Polyphonic Music. In Proceedings of the 12th International Society for Music Information Retrieval Conference, ISMIR 2011, Miami, FL, USA, 24-28 October 2011; pp. 233-238.

46. Chan, T.S.T.; Yang, Y.H. Complex and quaternionic principal component pursuit and its application to audio separation. IEEE Signal Process. Lett. 2016, 23, 287-291. [CrossRef]

47. Chan, T.S.T.; Yang, Y.H. Informed group-sparse representation for singing voice separation. IEEE Signal Process. Lett. 2017, 24, 156-160. [CrossRef]

48. Ramona, M.; Richard, G.; David, B. Vocal detection in music with support vector machines. In Proceedings of the 2008 IEEE International Conference on Acoustics, Speech and Signal Processing, Las Vegas, NV, USA, 31 March-4 April 2008; pp. 1885-1888.

49. Goto, M.; Hashiguchi, H.; Nishimura, T.; Oka, R. RWC Music Database: Popular, Classical and Jazz Music Databases. In Proceedings of the ISMIR 2002, 3rd International Conference on Music Information Retrieval, Paris, France, 13-17 October 2002; Volume 2, pp. 287-288.

50. Bittner, R.M.; Salamon, J.; Tierney, M.; Mauch, M.; Cannam, C.; Bello, J.P. Medleydb: A multitrack dataset for annotation-intensive mir research. In Proceedings of the 15th International Society for Music Information Retrieval Conference (ISMIR 2014), Taipei, Taiwan, 27-31 October 2014; Volume 14, pp. 155-160.

51. Hsu, C.L.; Jang, J.S.R. On the improvement of singing voice separation for monaural recordings using the MIR-1K dataset. IEEE Trans. Audio Speech Lang. Process. 2009, 18, 310-319.

52. Chan, T.S.; Yeh, T.C.; Fan, Z.C.; Chen, H.W.; Su, L.; Yang, Y.H.; Jang, R. Vocal activity informed singing voice separation with the iKala dataset. In Proceedings of the 2015 IEEE International Conference on Acoustics, Speech and Signal Processing (ICASSP), South Brisbane, QLD, Australia, 19-24 April 2015; pp. 718-722.

53. Maddage, N.C.; Wan, K.; Xu, C.; Wang, Y. Singing voice detection using twice-iterated composite fourier transform. In Proceedings of the 2004 IEEE International Conference on Multimedia and Expo (ICME)(IEEE Cat. No. 04TH8763), Taipei, Taiwan, 27-30 June 2004; Volume 2, pp. 1347-1350.

54. Vembu, S.; Baumann, S. Separation of Vocals from Polyphonic Audio Recordings. In Proceedings of the ISMIR 2005, London, UK, 11-15 September 2005; pp. 337-344.

55. Lukashevich, H.; Gruhne, M.; Dittmar, C. Effective singing voice detection in popular music using arma filtering. In Proceedings of the Workshop on Digital Audio Effects (DAFx'07), Bordeaux, France, 10-15 September 2007.

56. Forney, G.D. The viterbi algorithm. Proc. IEEE 1973, 61, 268-278. [CrossRef]

57. O'Shea, K.; Nash, R. An introduction to convolutional neural networks. arXiv 2015, arXiv:1511.08458.

58. Huang, H.M.; Chen, W.K.; Liu, C.H.; You, S.D. Singing voice detection based on convolutional neural networks. In Proceedings of the 2018 7th International Symposium on Next Generation Electronics (ISNE), Taipei, Taiwan, 7-9 May 2018; pp. 1-4. [CrossRef]

59. Gui, W.; Li, Y.; Zang, X.; Zhang, J. Exploring Channel Properties to Improve Singing Voice Detection with Convolutional Neural Networks. Appl. Sci. 2021, 11, 11838. [CrossRef]

60. Krause, M.; Müller, M.; Weiß, C. Singing Voice Detection in Opera Recordings: A Case Study on Robustness and Generalization. Electronics 2021, 10, 1214. [CrossRef]

61. Vu, T.H.; Wang, J.C. Acoustic scene and event recognition using recurrent neural networks. Detect. Classif. Acoust. Scenes Events 2016, 2016, 1-3.

62. Sutskever, I.; Martens, J.; Hinton, G.E. Generating text with recurrent neural networks. In Proceedings of the ICML 2011, Bellevue, WA, USA, 28 June-2 July 2011.

63. Vinyals, O.; Ravuri, S.V.; Povey, D. Revisiting recurrent neural networks for robust ASR. In Proceedings of the 2012 IEEE International Conference on Acoustics, Speech and Signal Processing (ICASSP), Kyoto, Japan, 25-30 March 2012; pp. 4085-4088. 
64. Hughes, T.; Mierle, K. Recurrent neural networks for voice activity detection. In Proceedings of the 2013 IEEE International Conference on Acoustics, Speech and Signal Processing, Vancouver, BC, Canada, 26-31 May 2013; pp. 7378-7382.

65. Olah, C. Understanding LSTM Networks. 2015. Available online: http://colah.github.io/posts/2015-08-Understanding-LSTMs/ (accessed on 10 November 2021).

66. Eyben, F.; Weninger, F.; Squartini, S.; Schuller, B. Real-life voice activity detection with lstm recurrent neural networks and an application to hollywood movies. In Proceedings of the 2013 IEEE International Conference on Acoustics, Speech and Signal Processing, Vancouver, BC, Canada, 26-31 May 2013; pp. 483-487.

67. Hochreiter, S.; Schmidhuber, J. Long short-term memory. Neural Comput. 1997, 9, 1735-1780. [CrossRef] [PubMed]

68. Ono, N.; Miyamoto, K.; Le Roux, J.; Kameoka, H.; Sagayama, S. Separation of a monaural audio signal into harmonic/percussive components by complementary diffusion on spectrogram. In Proceedings of the 2008 16th European Signal Processing Conference, Lausanne, Switzerland, 25-29 August 2008; pp. 1-4.

69. Cho, K.; Van Merriënboer, B.; Bahdanau, D.; Bengio, Y. On the properties of neural machine translation: Encoder-decoder approaches. arXiv 2014, arXiv:1409.1259.

70. Xingjian, S.; Chen, Z.; Wang, H.; Yeung, D.Y.; Wong, W.K.; Woo, W.C. Convolutional LSTM network: A machine learning approach for precipitation nowcasting. arXiv 2015, arXiv:1506.04214.

71. Lehner, B.; Widmer, G.; Sonnleitner, R. On the reduction of false positives in singing voice detection. In Proceedings of the 2014 IEEE International Conference on Acoustics, Speech and Signal Processing (ICASSP), Florence, Italy, 4-9 May 2014; pp. 7480-7484.

72. Paul, S.; Rao, K.S.; Das, P.P. Knowledge Distillation for Singing Voice Detection. arXiv 2021, arXiv:2011.04297. 